\title{
A Network-Based Virtual Reality Simulation Training Approach for Orthopedic Surgery
}

\author{
J. CECIL and AVINASH GUPTA, Oklahoma State University \\ M. PIRELA-CRUZ, Texas Tech University Health Sciences Center \\ PARMESH RAMANATHAN, University of Wisconsin-Madison
}

\begin{abstract}
The focus of this article is on the adoption of immersive and haptic simulators for training of medical residents in a surgical process called Less Invasive Stabilization System (LISS) plating surgery. LISS surgery is an orthopedic surgical procedure to treat fractures of the femur bone. Development of such simulators is a complex task which involves multiple systems, technologies, and human experts. Emerging Next Generation Internet technologies were used to develop the standalone on-line haptic-based simulator accessible to the students 24/7. A standalone immersive surgical simulator was also developed using HTC Vive. Expert surgeons played an important role in developing the simulator system; use cases of the target surgical processes were built using a modeling language called the engineering Enterprise Modeling Language (eEML). A detailed study presenting the comparison between the haptic-based simulator and the immersive simulator has been also presented. The outcomes of this study underscore the potential of using such simulators in surgical training.
\end{abstract}

CCS Concepts: $\bullet$ Computing methodologies $\rightarrow$ Modeling and simulation $\rightarrow$ Simulation types and techniques $\rightarrow$ Simulation by animation;

Additional Key Words and Phrases: Virtual reality, orthopedic surgery, medical simulation, Next Generation Internet technologies, immersive simulator

\section{ACM Reference format:}

J. Cecil, Avinash Gupta, M. Pirela-Cruz, and Parmesh Ramanathan. 2018. A Network-Based Virtual Reality Simulation Training Approach for Orthopedic Surgery. ACM Trans. Multimedia Comput. Commun. Appl. 14, 3, Article 77 (August 2018), 21 pages.

https://doi.org/10.1145/3232678

\section{INTRODUCTION}

In the recent years, various domains such as manufacturing, construction, design (among others) have utilized the Virtual-Reality-(VR)-based technologies for different purposes. In the field of medicine, the use of Virtual simulators for training purposes is increasing rapidly. Virtual simulators have been developed in a range of surgical fields such as heart surgery, laparoscopic surgery, and arthroscopic surgery among others [1-23]. The field of orthopedic surgery lags behind in adopting VR-based technologies; only a few Virtual simulators are available for orthopedic processes related to fracture treatment as most of the simulators focus on arthroscopic surgery.

This material is based upon work supported by the National Science Foundation [under grant number CNS 1257803]. Authors' addresses: J. Cecil, A. Gupta, M. Pirela-Cruz, and P. Ramanathan; emails: \{j.cecil, avinash.gupta\}@okstate.edu, cruzer@zianet.com, parmesh.ramanathan@wisc.edu.

Permission to make digital or hard copies of all or part of this work for personal or classroom use is granted without fee provided that copies are not made or distributed for profit or commercial advantage and that copies bear this notice and the full citation on the first page. Copyrights for components of this work owned by others than ACM must be honored. Abstracting with credit is permitted. To copy otherwise, or republish, to post on servers or to redistribute to lists, requires prior specific permission and/or a fee. Request permissions from permissions@acm.org.

(C) 2018 ACM 1551-6857/2018/08-ART77 \$15.00

https://doi.org/10.1145/3232678

ACM Trans. Multimedia Comput. Commun. Appl., Vol. 14, No. 3, Article 77. Publication date: August 2018. 
In this article, we discuss a network-based simulation environment for a specific orthopedic procedure called Less Invasive Stabilization System (LISS) plating surgery to treat fractures of the femur bone. The American Board of Orthopedic Surgery (ABOS) has also recognized the use of simulation-based training to improve surgical skills [24]. The objective of this article is to compare the haptic and immersive simulation environments and to recognize their potential as a training tool in the field of orthopedic surgery.

The developed simulators use two technology platforms (a) haptic technology and (b) immersive VR-based technologies. The immersive simulator uses immersive VR technology which has been used to design and build the training environments for LISS plating; immersion refers to a user being 'inside' (or immersed within) a simulator environment where the reference to the real world is removed. The immersive simulator was developed using the HTC Vive ${ }^{\mathrm{TM}}$ technology.

Several researchers have outlined the development of VR simulators in orthopedic surgery [25-47]. Haptic-based technologies allow a user to experience the sense of touch when interacting with a simulation environment; this has been investigated by several researchers in the context of medical surgical training $[48,50]$. Other research efforts have focused on developing simulators for surgical planning $[8,9,31,51-53]$. In orthopedic surgery, surgical planning refers to the identification of the major steps in that surgical process along with identifying which surgical instruments and tools need to be available for each of the surgical steps. In [37], a training simulator designed using Samsung Gear VR powered by Oculus is presented. The simulation showed the scenarios such as appropriate completion of primary survey, responding to vital cues from the monitor and recognizing fatal situations A study was conducted and the results indicated that the environment can be a useful medical training tool. Pre- and post-test method has been used to study the impact of using VR simulators developed for various medical surgical processes such as temporal bone surgery training [79], ureteroscopy [80] and endoscopy training [81].

Collaborative virtual environments enable distributed users to interact with each other [54-63]; it enables surgeons and residents to perform training activities in a collaborative manner from remote locations. Morris et al. [54] described a collaborative virtual environment for temporal bone surgery which used a private gigabit ethernet intranet. By the use of intranet, the voxel-based bone model was used for training interactions from both locations. A collaborative surgical system with a haptic interface is discussed in [55] which implemented a haptic based system in which the users could simulate surgical processes using virtual tools independently in two different locations. Paiva et al. [56] described SimCEC which is a cloud-based collaborative virtual environment for surgical education. A middleware platform was discussed in [52] for efficient collaboration using series of network management approaches such as service management, collaboration mechanisms, various deformation models, among others. A review of networking approaches in distributed surgical simulation indicates that while some approaches support distributed collaboration and remote access, they have not explored the adoption of Next Generation Internet technologies such as cloud computing and Software Defined Networking (SDN). SDN and cloud computing have been adopted in the network based approach presented in this paper. SDN not only reduces the complexity seen in today's networks, but also helps Cloud service providers host millions of virtual networks without the need for common separation isolation methods [64]. With the limitations of the current Internet, the Next Generation of Internet technologies are emerging which are being designed for low latency, high bandwidth, and distributed access. GENI-based next generation technologies adopt software-defined networking (SDN) principles. A key aspect of our approach is that the medical residents and students can access the simulator 24/7 and train on it from any location. An overview of this distributed interactive architecture and approach is provided in Section 2.2.

Only a few research efforts [65-67] have adopted a user-centered design approach. A requirements specification process has been described [65] for a maxillo-facial surgery simulator where 
they focused on analyzing the surgical tasks from expert's point of view. In [66], a user-centered design process for the development of an orthopedic surgical robot has been described in which information was gathered from questionnaires and workshops with domain experts. A user-centered design approach following the ISO guidelines for the development of a bone surgery simulator was described [67]. While these articles focus on user-centered approaches, they do not emphasize the importance of information modeling. Information modeling is a well-accepted software engineering practice; information models (activity or use case diagrams) capture the complexity of the target process for which the software system has been designed. In our approach, we have developed information models based on close and continuous interactions with the surgeons which enabled a better understanding of the complex relationships by the simulator design and development team within the surgical process.

There has been a lesser emphasis in the reported literature on research relating to the adoption of information models and work-flow-based techniques in the design and development of surgical simulators. One such approach [68], involves the use of a Work-flow Integration Matrix (WIM) in the design of surgical information systems. WIM uses theories of human behavior in problem solving and investigates the role of evidence-based decision-making for the development of new surgical technologies. In [69], Jannin highlighted the need for process models in computer-assisted surgery. One of the other methods used in the modeling of surgical tasks is Hierarchical Task Analysis (HTA) [70]. However, the HTA approach does not model or capture key attributes such as information or physical inputs, and constraints; modeling such attributes is necessary to obtain a better understanding of functional and process relationships which in turn enables a stronger foundation that is necessary to build a virtual environment.

The design of the simulators discussed in this article addresses the modeling of such attributes using an information modeling approach. The information modeling approach used encompasses the major elements of process modeling including identifying the information inputs, constraints, performing mechanisms as well as the intermediate decision outcomes of the various sub-tasks; by modeling such relationships as well as explicitly capturing temporal precedence constraints, it provides a more fundamental basis to understand complex surgical processes which subsequently provides a structured foundation to design and develop a surgical simulator; the complexity of a given surgical process is further modeled by levels of decomposition which provides an effective way to model the process and system level interactions involving software, equipment/tools and humans involved. The information modeling approach used in the design and development of the Virtual simulators is described in our previous articles [71, 72].

The focus of this article is to compare and study the effectiveness of using haptic and immersive platform-based simulators in orthopedic surgical training.

\section{ARCHITECTURE OF THE SIMULATION ENVIRONMENTS}

The training simulators (haptic and immersive) have several modules; an introduction module provides an 3D overview of the surgical simulation in a step by step manner without much user interaction (except for starting, pausing and stopping the simulation of the entire procedure). Subsequently, the users (or residents) interact with training modules and practice the various surgery steps using the haptic device for the haptic-based simulator and using handheld controllers for the immersive simulator. Additional details of these environments and interactions are presented in Section 2.5.

Both training simulator platforms were built using C\#, JavaScript, and the Unity game engine on the Windows platform. The various virtual objects in the simulation environment such as various bones; surgical implants such as LISS plates and supports; and surgical tools, tables, and other objects were built using Solidworks ${ }^{\mathrm{TM}}$. The bones were built after studying MRI images of patients' 


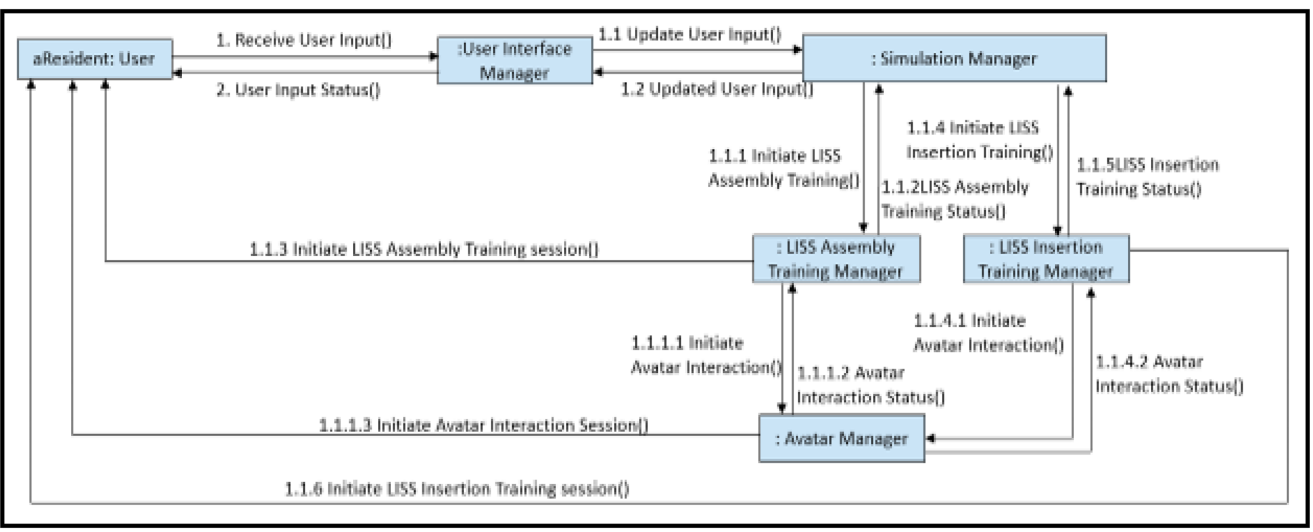

Fig. 1. A partial view of communication diagrams used as part of the process to build the simulators.

bones and consulting with expert surgeons. In the current implementation, only adult bone models were created to support the training. An expert team of three surgeons validated the correctness of various bones, instruments, tool,s and other entities in the surgical environments.

In Section 2.1, the design of the simulation environments using Unified-Modeling-Language(UML)-based communication diagrams is presented. A discussion of standalone and on-line haptic based simulator is presented in Section 2.2. In Section 2.3, the Vive-based immersive environment is discussed. The modeling of the LISS plating surgical process using eEML modeling language is described in Section 2.4. In Section 2.5, the six training environments developed based on the LISS plating surgical steps are discussed.

\subsection{The Simulation Manager}

The simulation manager is a software component that coordinates interactions between the various environments and the user. UML-based communication diagrams have been used to show the interactions between various software components. In the field of software engineering, the Unified Modeling Language (UML) is one of the leading modeling language used in the software engineering process (to build software systems, in general). Communication diagrams (within UML) are design representations that model the interactions between various software components or modules within a system [73]. The various training environments in the simulation are coordinated by software entities named managers (as shown in Figure 1); As indicated in the communication diagram (Figure 1), the simulation manager coordinates the simulation-based training activities through interactions with the managers of each of the training components and the avatar manager. The avatar manager coordinates interactions between users and simulators with the help of avatars; an avatar (as shown in Figure 10) is a 3D humanoid model that serves as a cyber assistant and helps the residents with the training activities interactively (using both voice-based guidance as well as text-based descriptions). These environments and manager components were implemented using C\# and JavaScript. The simulation manager and other software components are described in more detail in [74].

\subsection{Haptic-Based On-Line Simulator}

The haptic-based simulator utilizes the haptic interface which is provided using the Geomagic Touch $^{\mathrm{TM}}$ device; it allows users to touch, grasp, and interact with various surgical tools during the simulation activities. The haptic interface primarily functions to give an intuitive "feel" for various tasks (such as picking up various plates or tools, placing them accurately in a certain location, etc.). 


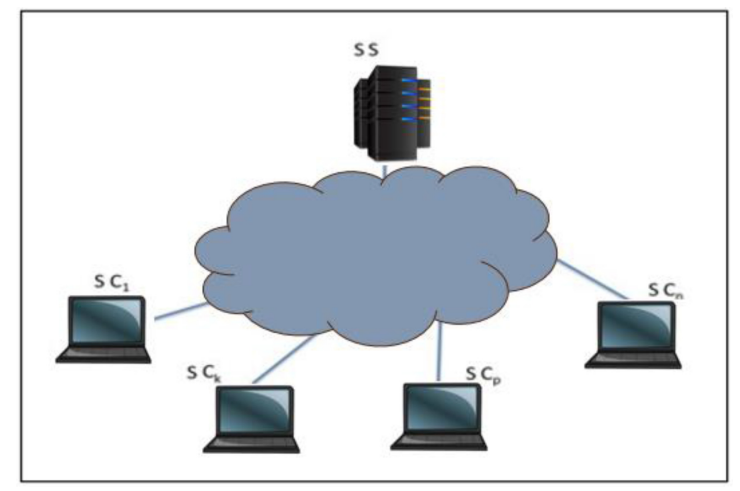

Fig. 2. Multi-participant simulation architecture in Unity. (SS is the simulation server, and SCs are the simulation clients.)

The haptic device has a stylus with two buttons (dark and light colors): A resident can pick up a virtual instrument or any other object in the simulation environment by pressing and holding the dark-colored button which allows the user to make "virtual" contact with a target object while moving a grasped object to a target destination.

The physics-based simulation of the drilling activities is based on [32]. For the pickup/placement of various plates, the surgeons wanted the residents to feel "contact" when they picked up or positioned a plate as well to provide a basic feedback when one plate collided with another part. As part of the requirements, they did not want an accurate physics-based modeling as long as the users felt there was some type of a collision during these surgical training sessions); for this reason, physics-based modeling was not incorporated during the pick and place simulation tasks in the training environment (with the exception of the modeling of the drilling activities); a basic collision detection feature was incorporated to enable residents to feel contact when plates collided or touched each other (or other objects such as the jig or another tool during the training).

For the on-line training activities, Next Generation Internet technologies have been implemented to allow residents and users access the simulator 24/7 from various locations. While the current Internet has become ubiquitous in supporting novel societal and commercial usages, it was never originally designed for such a wide range of applications [59]. The incremental solutions used to address these concerns can be viewed as short-term "patches"; further, the range of applications has necessitated hundreds of additional protocols and extensions, which make its management more and more complex. It has also posed demanding technological and policy challenges in security, mobility, heterogeneity, and complexity [75]. For these reasons, several initiatives have started the development of the Next Internet in the US, Europe [76], Japan and other countries [76]. In the U.S., the Global Environment for Network Innovations (GENI) $[75,77]$ is an initiative led by the National Science Foundation that focuses on the design of the Next Generation Internet frameworks based on advanced networking characteristics which include multi-gigabit bandwidth, low latency, software-defined networking and/or control over geographic location of resources.

To support this on-line training approach, the surgical training application has been built on top of a 3D Virtual Reality engine called Unity. The Unity engine used in the simulator is a popular and easy-to-use platform for simulation and other gaming applications. Figure 2 shows the multiparticipant architecture in Unity. Simulation Server (SS) in Figure 2 refers to the meeting place that puts simulation instances in touch with the Simulation Clients (SC) who wants to connect 
with it. As the Unity based architecture is susceptible to single point failure of the SS, if the SS fails and/or if the network connection to the SS fails, the entire system fails and all SCs are deregistered and disconnected. This may be acceptable in the context of other multi-participant applications, but it is not acceptable in the surgery context given the sensitive nature of the surgical training application.

In the surgical application context, the participants are the trainees (students and residents). They can be also referred to as Tele-Medicine Clients (TMCs). Since Unity is not an open-source platform, it is not possible to modify its libraries to support resiliency against connection failures to the Tele-Medicine Server (TMS). As such support is important in telemedicine applications, Software-Defined Networking (SDN) principles have been used to increase resiliency to TMS failures. SDN separates network control and forwarding function which enables network control to be directly programmable which makes it ideal for today's dynamic and high-bandwidth applications. Some of the benefits of SDN are that it is directly programmable, agile, centrally managed, and open standards-based and vendor-neutral [78].

Figure 3 shows the SDN-integrated architecture of our surgical application. There are r redundant TMSs in this architecture. Failure to connect to up to $r$-1 TMS can be seamlessly tolerated in this networked architecture. To achieve this, the TMCs do not connect directly to a TMS. Instead, each TMC connects to the TMSs through proxies implemented by SDN switches (realized through OpenFlow); OpenFlow is a SDN standard which allows network controllers to decide the network path packets across the network of switches. If there are $m$ Open Flow Proxies (OFPs), then the TMCs are partitioned into $m$ groups, and each group connects to the TMSs through one of the OFPs. The OFPs play a crucial role in providing failure resiliency without introducing much latency.

\subsection{Development of the Immersive Training Simulator}

The immersive simulator was built using the HTC Vive, which is one of the recent low-cost platforms and was built using the Steam VR tool kit [82] and a third party Virtual Reality Tool Kit (VRTK) [83]. The Vive headset provides a $110^{\circ}$ field of view [84] and has two wireless handheld controllers and two cameras (Figure 4) to track the position of the user in the training environment. The immersive simulator is a standalone simulator in which the users get a realistic view of the training environments through the level of immersion provided by the Vive. A user can be seen interacting with one of the training environments in Figure 4.

\subsection{The LISS Plating Training Environments}

Both the haptic and immersive simulators consist of six training environments built to train the residents. The environments include the following: assembling the LISS plate, inserting the LISS plate, position training, reducing the fracture, screw insertion, and guide removal processes. These six training environments are based on the LISS plating surgical steps. In the LISS Plate Assembly Training Environment, the user assembles the LISS plate with the help of the insertion guide and bolts. After the assembly is complete, the user inserts the plate inside the patient's leg in LISS Insertion Training Environment. In Position Training Environment, the user positions the plate correctly inside the leg. The user reduces the fracture in the Fracture Reduction Training Environment. After the fracture is reduced, the user attaches the plate in the femur bone using screws in Screw Insertion Environment. Finally, the insertion guide is removed in the Guide Removal Training Environment. To successfully complete these training steps, various tools are required which include Insertion Guide, Fixation Bolt, Stabilization Bolt, Insertion Sleeve, Pull Reduction Instrument, Trocar, Locking Screws, Screw Driver Shaft, and Power Tool. Each training environment has two modes: (i) practice and (ii) test. 


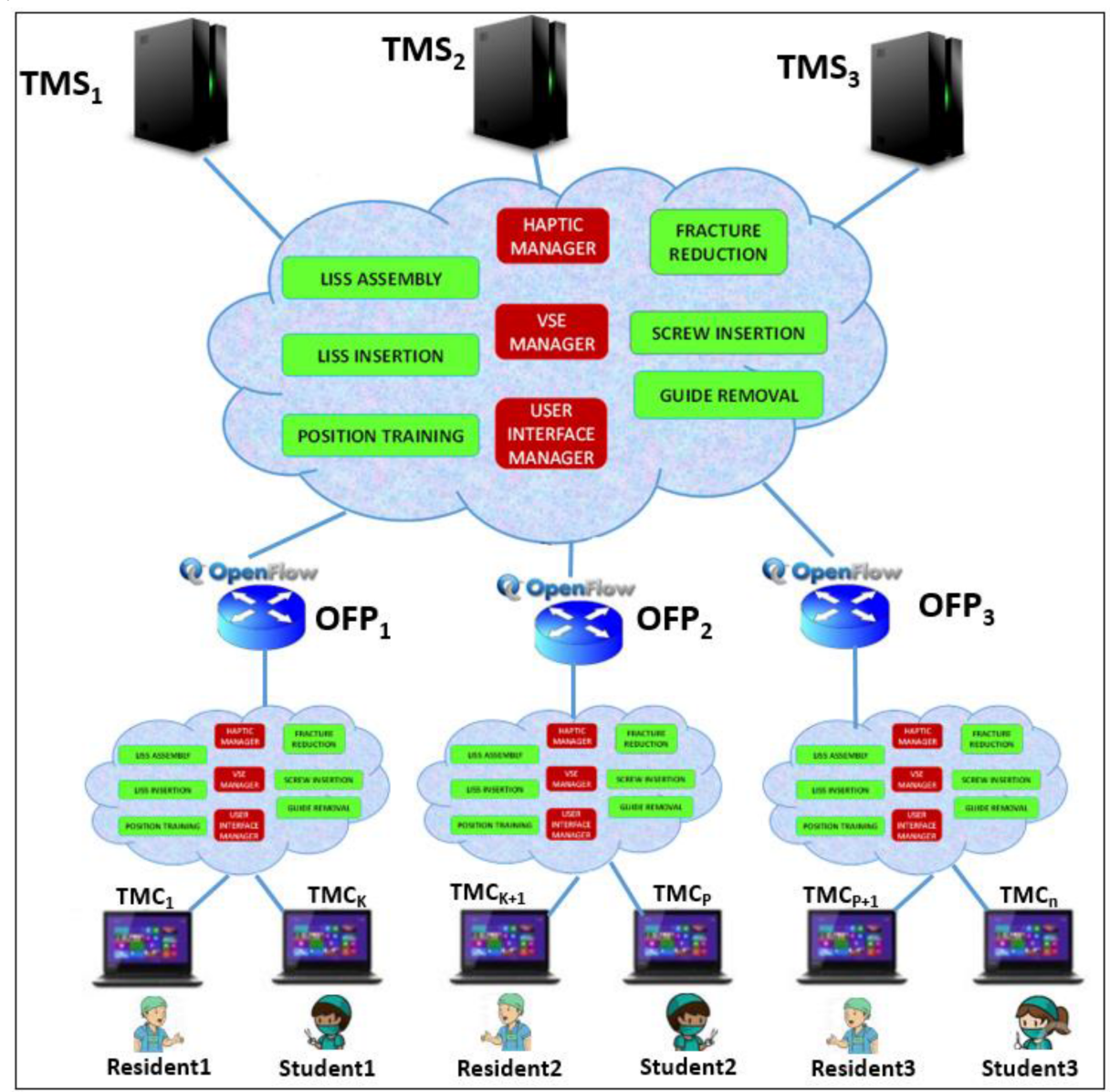

Fig. 3. Architecture for the telemedicine surgical application. (TMS is the telemedicine server, TMCs are telemedicine clients, and OFPs are OpenFlow switch-based proxies.)

In practice mode, text-based cues (shown in Figure 5) and audiovisual cues (using humanoid avatar as shown in Figure 10) were provided to the user to perform the training. The expert surgeons indicated they wanted such audiovisual and text cues during the practice sessions.

Further, a "snap on" feature was included which makes it easier for the residents to assemble parts and position them correctly. When the user is close to the final position of a part during assembly, the "snap on" feature snaps the part in the correct position and orientation as shown in Figure 5. A continuous simulation of the part moving from the initial position to the final position is also provided in the practice mode (Figure 5). It helps the users to follow the correct path while performing the assembling or positioning processes.

The test mode is used to test the skills after the residents complete training sessions in the practice mode. In this mode, the text/voice- based cues and snap-on features are removed from the training environment.

In this article, four of the six training environments namely LISS Plate Assembly Training Environment, LISS Insertion Training Environment, Fracture Reduction Training Environment, and 


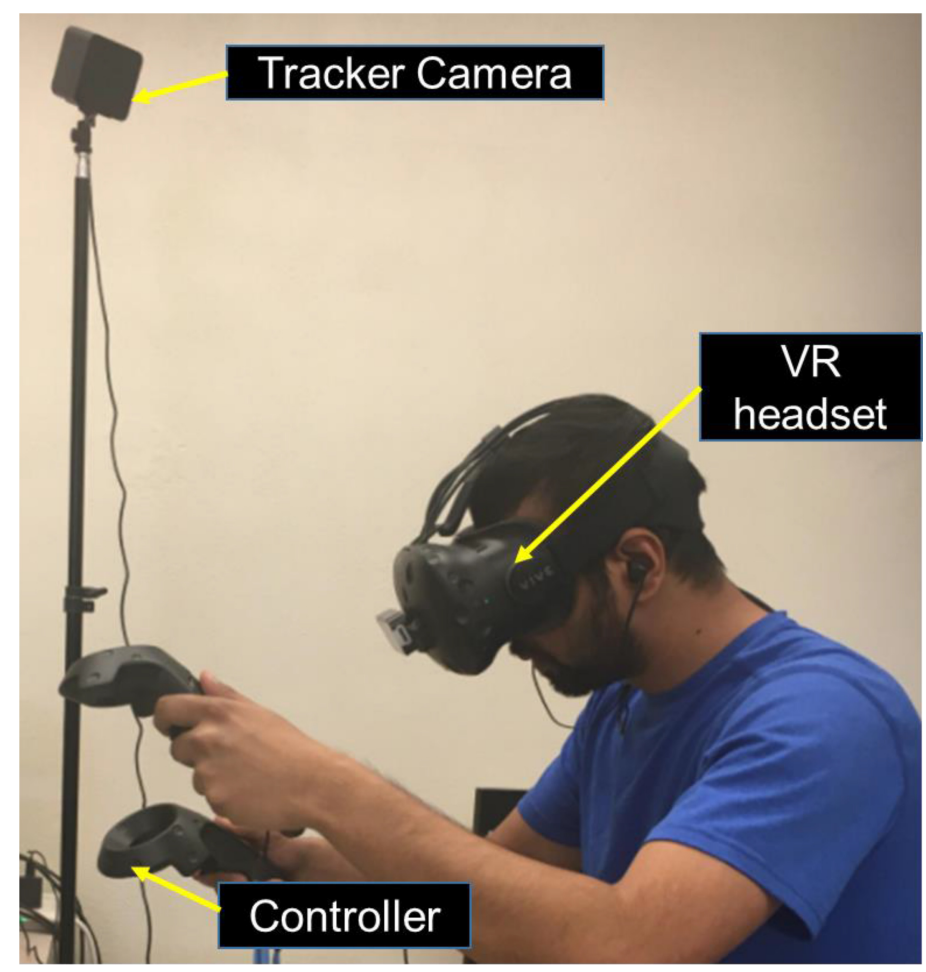

Fig. 4. A user interacting with the training environment using the Vive.

Guide Removal Training Environment are discussed in detail. A discussion of the Position Training Environment and Screw Insertion Environment is provided in an earlier article [74].

2.4.1 LISS Plate Assembly Training Environment. The LISS Assembly Training Environment focuses on helping the residents to become familiar with assembling the LISS plate from the necessary components. In general, this process is a sequence of key steps which include the assembly of LISS Insertion Guide and components (see Figure 6(a)), followed by insertion of the fixation bolt through the appropriate distal hole in the insertion guide attachment (see Figure 6(b)); this is followed by the insertion of a stabilization bolt with insertion sleeve which stabilizes the LISS plate and the guide (see Figure 6(c)). As a user progresses through the training activities, the simulation manager updates a state chart that provides a snapshot of the progress of the training activities during the design of the simulators, these key steps were identified as process entity elements; the main simulation outcome that the simulation manager tracks and monitors after each training step in this environment is identified as a Decision Outcome.

In Figure 6, the views of the LISS Assembly Training Environment within the haptic-based simulator is provided. In the haptic-based simulator, the user picks up the surgical implants and tools by pressing the button showed in Figure 7. To place the part in the required position, the user releases the button. A view of the environment as seen by the user wearing a Vive headset using the immersive simulator is provided in Figure 8. In the immersive environment, the user picks up the parts by pressing the side button on the handheld controller as shown in Figure 9 . The user releases the side button when the part is ready to be placed in the desired position. 


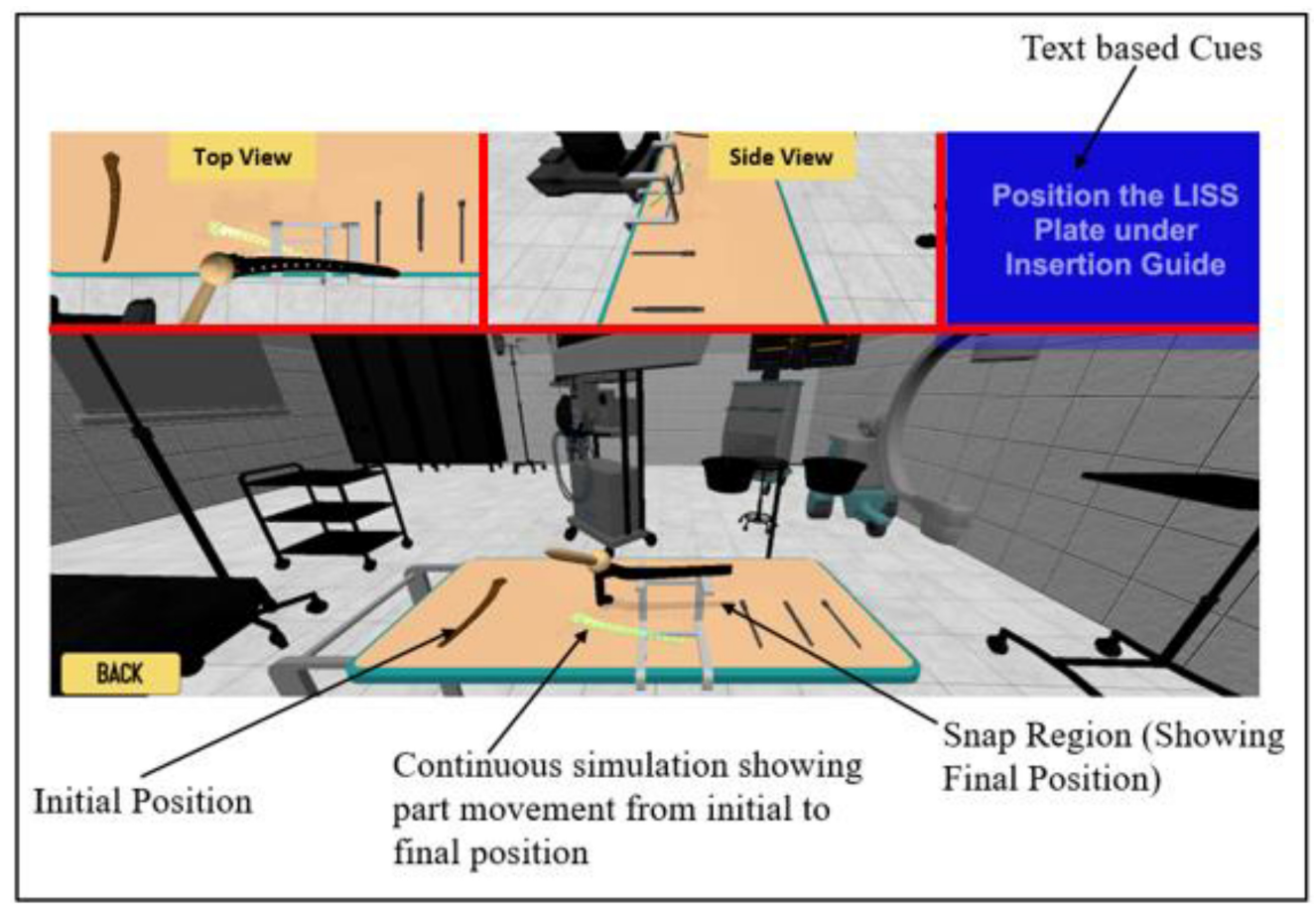

Fig. 5. Multiple-view-based training scenario of LISS plate assembly environment.

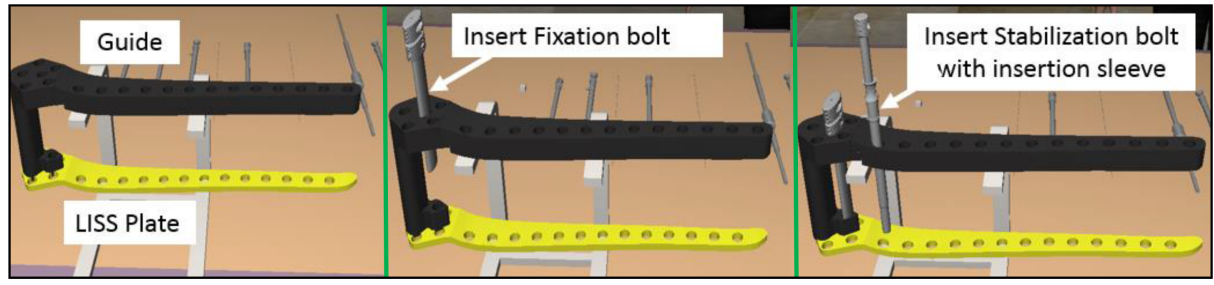

Fig. 6 (a), (b) and (c). Views of some of the steps in the LISS Assembly Training Environment in the hapticbased simulator.

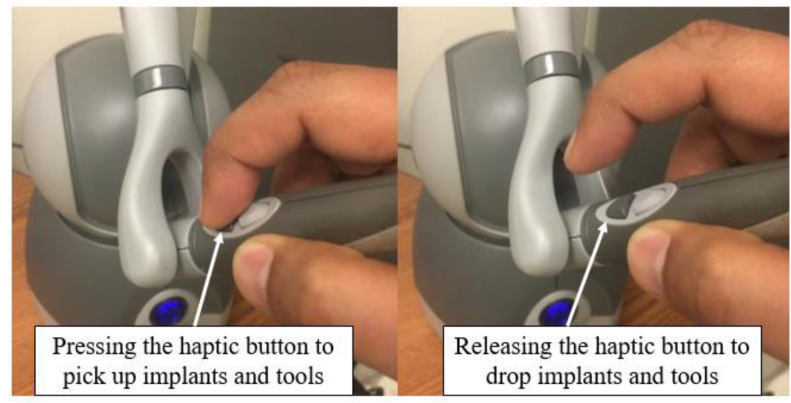

Fig. 7. A user interacting with simulator using the haptic device. 


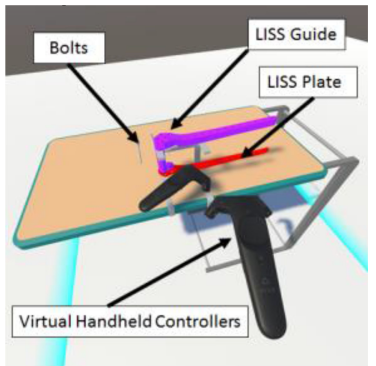

Fig. 8. The view of the LISS Assembly Training Environment as seen by the user wearing the Vive headset.

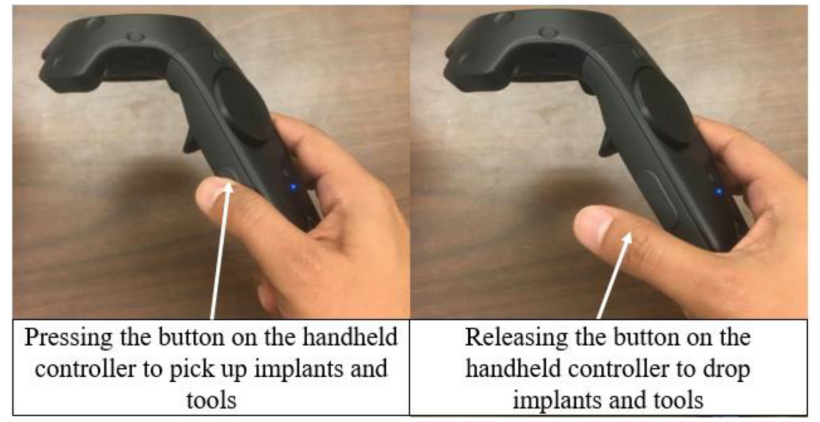

Fig. 9. A user interacting with simulator using the handheld controller.

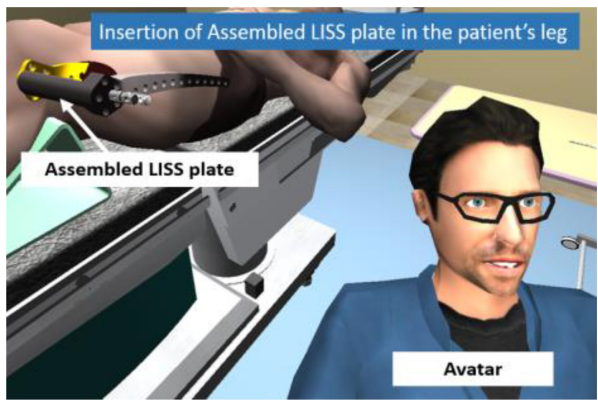

Fig. 10. A view of the haptic-based LISS Insertion Training Environment.

2.4.2 The LISS Insertion Training Environment. The training objective of the LISS insertion environment is to practice the inserting of the assembled LISS plate (from the previous step) between the vastus lateralis and the periosteum muscle (using a LISS insertion guide). Several important principles are emphasized including plate positioning and orientation, as well as aligning/positioning the plate with respect to the distal femur. The plate (shown in Figure 10) is inserted using incisions in the proximal and distal side of the bone. Residents are encouraged to pause and study the positioning and orientations of the components involved and surgical tools. Figure 10 shows a view of haptic-based LISS Insertion Training Environment. In Figure 11, a view of the immersive LISS Training Environment is shown.

2.4.3 Fracture Reduction Training Environment. The training objective of this environment is to help the residents practice and become skilled in fracture reduction using the pull reduction 


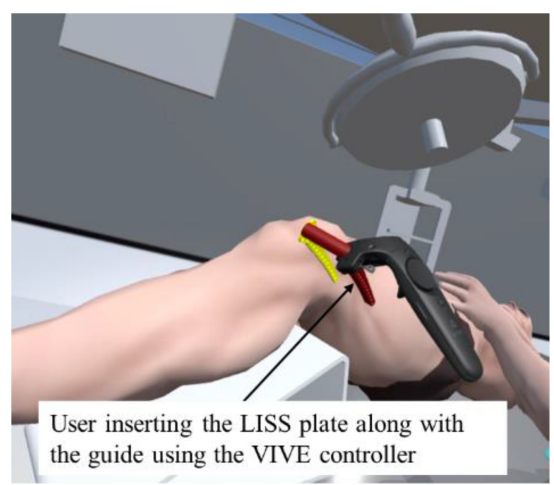

Fig. 11. A view of the immersive LISS Insertion Training Environment.

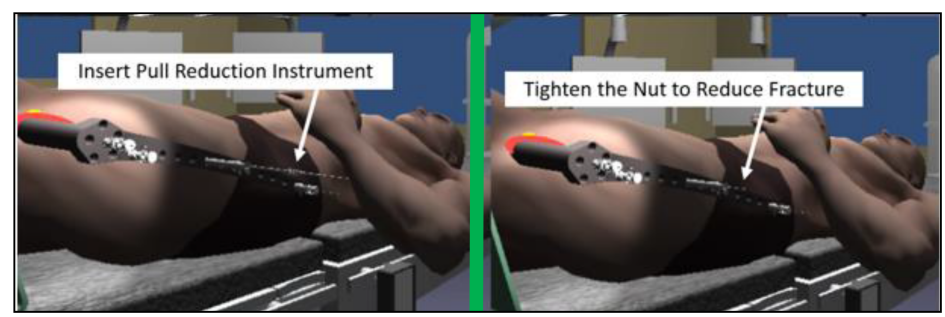

Fig. 12. View of the Fracture Reduction Training Environment for the haptic simulator.

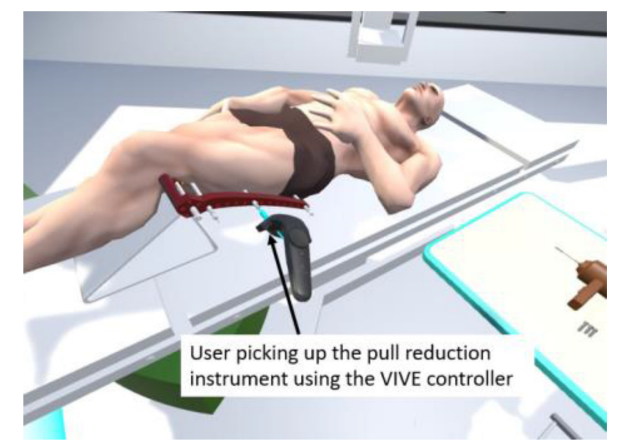

Fig. 13. A view of the Fracture Reduction Training Environment for the immersive simulator.

instrument. The pull reduction instrument helps restore the fractured bones to the correct position and alignment. During training, images from the haptic simulator for two of the steps in this process is shown in Figure 12. The image from the immersive simulator for the Fracture Reduction Training Environment is shown in Figure 13.

2.4.4 Guide Removal Training Environment. In this last training environment, the residents practice the removal of the insertion guide and other components. Initially, the insertion sleeve and stabilization bolt in the LISS insertion guide are removed from the LISS plate. Subsequently, the guide with fixation bolt is removed at the end. A user interacting with the environment to remove the insertion guide using haptic device is shown in Figure 14. In Figure 15, a user is seen removing the insertion guide using the Vive controller. 


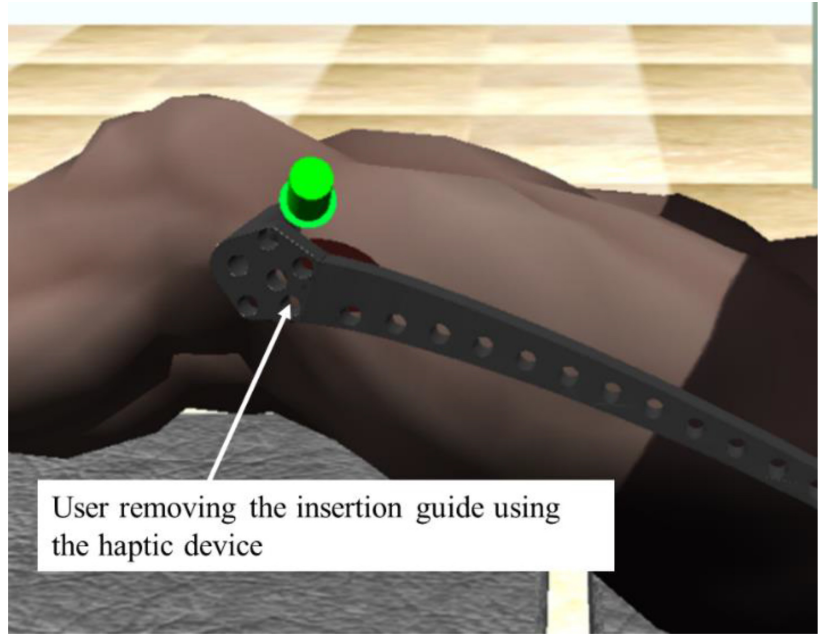

Fig. 14. A user interacting with haptic-based Guide Removal Training Environment.

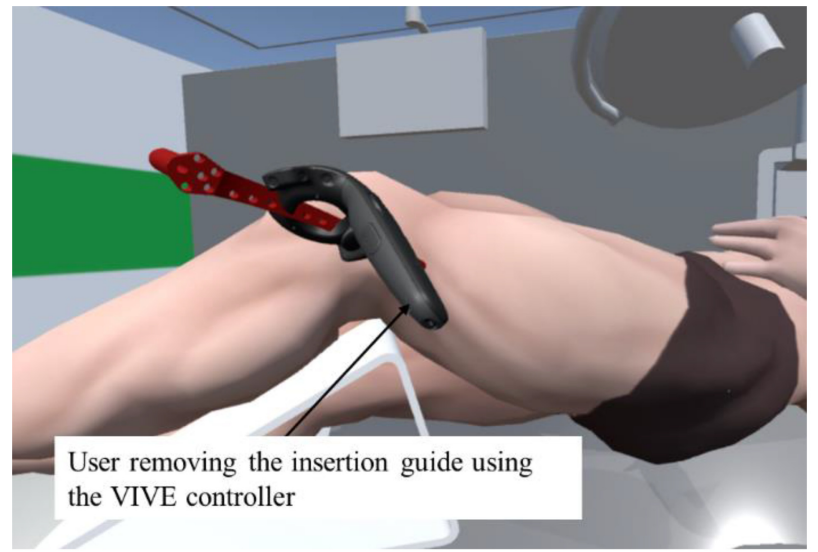

Fig. 15. A user interacting with Vive-based Guide Removal Training Environment.

\section{LEARNING STUDIES AND DISCUSSION}

\subsection{Role of Expert Surgeons in Design and Development of the Simulator}

The role of expert surgeons has been crucial in the design and development of the surgical simulators. A summary discussion of their roles in different phases of the project follows.

Roles in Planning and Information Modeling

(1) Helped identify data inputs, control factors and resource identification which were key to creation of the information model discussed.

(2) Elaborated on the details of each surgical step and how it's performed along with the functional objective of each surgical step.

(3) Listed the expected training outcomes (or areas of proficiency expected after the training). 
(4) Identified areas of skills or situations where residents can tend to make mistakes (due to complexity in a procedure).

\section{Roles in Simulator Building or Development Phase}

(1) Verified correctness of surgical procedures and steps by interacting with all modules and environments as they were built (along with providing modifications and corrections to the simulation details).

(2) Gave suggestions on improving voice and text cues within each simulation module.

\section{Roles in Post-Development Phase}

(1) Discussed the types of learning studies to be conducted and developed questionnaires for the assessment of learning.

(2) Coordinated learning interactions with residents.

\subsection{Learning Interactions}

The usefulness and impact of using the simulators as an educational and training resource were studied through interactions with surgeons, residents, and medical students at the Paul L. Foster School of Medicine, Texas Tech University Health Sciences Center in El Paso, Texas. Prior to the interaction with the residents and students, the lead expert surgeon in this project (Dr. M. Pirela-Cruz) along with two other surgeons interacted with the various simulation scenarios and training environments to ensure their correctness and provided detailed feedback on various intricacies that needed to be reflected during various training scenarios; this resulted in various changes to the surgical simulation content and training aspects. After these changes were implemented and verified, the learning studies involving medical residents and students was undertaken. The learning studies were conducted in multiple phases for 2 years with a gap of 6 months between each phase.

Two types of assessments were conducted: (1) the performance of the GENI-network-based haptic simulator (in terms of latency) was studied when users accessed it remotely, and (2) the impact on learning between using the standalone (not network-accessed) haptic and immersive simulator. In an earlier work, an assessment of learning outcomes involving residents being trained with the help of surgeon using a network-based collaborative haptic simulator has been discussed in one of our previous articles [74].

We conducted both quantitative and qualitative assessments related to the impact on learning using these simulators. Additional details about these learning assessments follow in 3.2 .1 and 3.2.2.

3.2.1 Quantitative Assessment (using Pre- and Post-Test). In the quantitative assessment of learning, a pre-test was first conducted for all the participants to test their knowledge/skills relating to LISS surgery. After the pre-test, the participants interacted with the simulator for a total of sixty minutes in which they were allowed to practice and learn about the LISS plating surgical processes as many times as they needed. Subsequently, the participants were evaluated through a post-test. A total of ten questions were asked in the pre-test and the same questions were repeated in the post-test as well. The three expert surgeons scored all the tests. The answers to the questions were not provided to the participants between the pre- and the post-tests. The scores of the pre- and post-tests were only released after the post-test was completed. The improvement is score (post-test score - pre-test score) was categorized in two levels by the three expert surgeons. The improvement of over 40 points was considered to be significant by the 
Table 1. Learning Interactions Demographics

\begin{tabular}{|l|l|c|c|}
\hline & & Students & Residents \\
\hline \multirow{2}{*}{ Control Group } & on-line Haptic Simulator & 6 & 4 \\
\cline { 2 - 4 } & Comparison Study & 12 & 12 \\
\hline \multirow{3}{*}{ Study Group } & on-line Haptic Simulator & 22 & 10 \\
\cline { 2 - 4 } & Comparison Study: Haptic Simulator & 12 & 12 \\
\cline { 2 - 4 } & Comparison Study: Immersive Simulator & 12 & 12 \\
\hline
\end{tabular}

Table 2. Pre- and Post-Test Results of Control Group and Haptic On-Line Simulator

\begin{tabular}{|c|c|c|c|c|}
\hline & \multicolumn{2}{|c|}{ Control Group } & \multicolumn{2}{c|}{ on-line Simulator } \\
\hline & Pre-Test & Post-Test & Pre-Test & Post-Test \\
\hline Mean & 14 & 15 & 28.43 & 58.43 \\
\hline $\mathrm{n}$ & 10 & 10 & 32 & 32 \\
\hline
\end{tabular}

surgeons. If the participants showed an improvement of 10-40 points, it was considered to be moderate. The following are the ten questions asked in the pre- and the post-tests:

- How does one ensure alignment of the plate with the holding apparatus?

-What device positions the extremity correctly on the operating room table?

-What x-ray projections are most important to obtain during surgery?

-What potential complication can occur if the plate is positioned too distal?

-What device is used for the initial insertion of the screws?

-Which is the last bolt to be removed? and why?

-Which portion of the plate is applied on the condyles of the femur?

-What instrument or tool is used to apply the plate to the jig?

- How many critical radiographic views are needed for LISS plating of the distal femur?

-Which portion of the plate is applied on the shaft of the femur?

On-Line Haptic Simulator Study. Thirty-two participants performed learning studies on the online haptic simulator. Among the 32 participants, 22 were medical students and 10 were residents as shown in Table 1. The pre- and post-test results for the on-line simulator are shown in Table 2. Twenty-seven out of 32 participants demonstrated improvements in their understanding of the LISS plating surgical process. Out of the 27 students who demonstrated improvement, 12 students showed significant improvement (40 points and above) and 15 students performed at a level termed moderate improvement (10-40 points). The average score for all the participants in pre-test was 28.43. There was a significant improvement (58.43) in the average score of the participants after interacting with the simulator. These results underscore the impact of using Virtual simulators to improve the understanding of orthopedic surgery procedures. Feedback was also obtained from the quantitative analysis test regarding the usefulness of the haptic interface and the teaching avatar used in the simulator. Nearly $80 \%$ of participants indicated that the use of haptic interface in the simulator helped them gain a better understanding of the LISS surgical process. About $85 \%$ of the participants indicated that the teaching avatar helped them in interacting with the simulator.

Comparison Study between Haptic and Immersive Simulators. To compare the haptic and immersive simulators, an assessment was conducted involving three groups (A, B, and X) of medical students/residents. The demographics of the participants is shown in Table 1. Group A was exposed to training activities using the haptic simulator. Group B was trained using the immersive 
Table 3. Pre- and Post-Test Results of the Control Group and Haptic and Immersive Simulator

\begin{tabular}{|c|c|c|c|c|c|c|}
\hline & \multicolumn{2}{|c|}{$\begin{array}{c}\text { Control Group } \\
(\mathbf{X})\end{array}$} & \multicolumn{2}{|c|}{$\begin{array}{c}\text { Haptic-Based Simulator } \\
\text { (A) }\end{array}$} & \multicolumn{2}{c|}{$\begin{array}{c}\text { Immersive Simulator } \\
\text { (B) }\end{array}$} \\
\hline & Pre-test & Post-test & Pre-test & Post-test & Pre-test & Post-test \\
\hline Mean & 10.41 & 11.25 & 13.33 & 60.83 & 12.5 & 60.41 \\
\hline $\mathrm{n}$ & 24 & 24 & 24 & 24 & 24 & 24 \\
\hline
\end{tabular}

Table 4. Experimental Design for the ANOVA Test

\begin{tabular}{|l|l|c|c|}
\hline \multicolumn{2}{|c|}{} & \multicolumn{2}{|c|}{ Virtual Environment } \\
\cline { 3 - 4 } \multicolumn{2}{|c|}{} & $\begin{array}{c}\text { Haptic-based } \\
\text { Simulator }\end{array}$ & $\begin{array}{c}\text { Fully immersive } \\
\text { Simulator }\end{array}$ \\
\hline \multirow{2}{*}{ Assessment Type } & $\begin{array}{l}\text { Pre- and Post-Test Score } \\
\text { Difference }\end{array}$ & DV & DV \\
\cline { 2 - 4 } & Control & DV & \\
\hline
\end{tabular}

simulator. Group X, the control group, was not exposed to training activities on any simulator. The primary objective was to study the effectiveness of using a haptic interface for training versus a non-haptic but immersive environment for training. Each group consisted of 12 participants who had not interacted with any of the simulators before. Groups A and B participated in a pre-test; subsequently, one group was trained using the haptic simulator and the other group was trained using the immersive simulator; post-tests were subsequently conducted. The training period was 60 minutes on both the simulators. Group X performed the pre-test, waited for 60 minutes without any interaction, and performed the post-test. The results of these pre- and post-tests are shown in Table 3.

All the participants in groups A and B showed significant learning improvements in the post-test whereas the learning improvements for control group $\mathrm{X}$ was minimal. The average improvement in the group trained using the immersive simulator was 47.91 and the group trained using the haptic simulator was 47.5. Twenty out of 24 participants from group A showed significant improvement (40 or above) and 4 participants showed moderate improvement (10-40 points). For group B, 21 out of 24 participants showed significant improvement and only 3 participants showed moderate improvement.

The pre- and post-test scores range from 10.41 to 60.41 (these scores are the average scores for all the participants). It can be seen from the results that the pre-test scores range from 10.41 to 13.33. The range of scores in these pre-tests indicate that some of the participants may had prior understanding of the LISS plating surgical process.

We performed an ANOVA to test if there is a difference of mean scores between the three groups (A, B, and X). The difference between pre- and post-test scores were assigned as a dependent variable (as seen in Table 4) for all three groups (the control group, the haptic-based simulator, and the immersive simulator). The $\mathrm{F}$ value from our test is significantly higher than the critical F value $(86.95>3.12)$, as seen in Table 5 , which indicates that there is a statistically significant difference in the mean scores between the control group and groups A and B.

3.2.2 Qualitative Feedback Surveys. After the completion of the comparison study, a Qualitative Feedback Survey (QFS) was also conducted. QFS surveys are also presented in [72] for standalone (not the on-line) haptic simulator based learning interactions. The 48 participants 
Table 5. Results from the ANOVA Test

\begin{tabular}{|l|r|r|r|r|l|l|}
\hline SUMMARY & & & & & & \\
\hline Groups & Count & Sum & Average & Variance & & \\
\hline control Group & 24 & 70 & 2.916666667 & 47.64492754 & & \\
\hline vive & 24 & 1310 & 54.58333333 & 399.8188406 & & \\
\hline Haptic & 24 & 1140 & 47.5 & 202.173913 & & \\
\hline & & & & & & \\
\hline & & & & & & \\
\hline ANOVA & SS & $d f$ & MS & F & P-value & F crit \\
\hline Source of Variation & 37658.33333 & 2 & 18829.16667 & 86.95231456 & $1.39 \mathrm{E}-19$ & 3.129644 \\
\hline Between Groups & 14941.66667 & 69 & 216.5458937 & & & \\
\hline Within Groups & & & & & & \\
\hline & 52600 & 71 & & & & \\
\hline Total & & & & & & \\
\hline
\end{tabular}

(who interacted with haptic and immersive simulators during the comparison study) participated in this survey. The participants rated both the simulators on 13 criteria and provided a score between 1-10 (10 being the highest and 1 being the lowest). A few of these criteria (7-12) were acquired from NASA Task Load Index (TLX) [49] which is considered a leading standard to measure subjective workload for users in various applications ranging from simulators to aircraft cockpits. Table 6 shows the average score for each criterion for both haptic and immersive simulators. Based on this survey, it can be noted that participants found using the immersive simulator more effective, user friendly and easier to interact than using the haptic simulator. The text-based support was more useful in the haptic-based simulator as it was easy to view them on the 2D screen whereas the avatar-based support was more suited for the immersive 3D environment of the Vive-based simulator. The other measures were comparable for both the simulators.

Performance of GENI Network. During the learning interactions on the on-line haptic-based simulator, performance of the GENI-based network with respect to latency was also studied. An Internet Control Message Protocol (ICMP) ping was used to measure the network latency.

Table 7 shows the latency data when the on-line Haptic simulator was used simultaneously by two and three users from different locations (the latency was measured between the user's computer and the server). The latency data was collected for a 2-hour period. The results from the test show that the average latency is stable around 48 milliseconds (which is an acceptable measure).

Other researchers have conducted transfer validity studies to study the transferability of skills from one system to another [85, 86]; this involves determining if the skills learnt during training using the VR simulator are transferable to a physical simulator. As the transfer validation is beyond the scope of the current phase of this project, we plan to conduct such transfer validity studies in the next phase of the project.

\section{CONCLUSION}

In this article, the focus of the discussion was on the comparison of haptic-based and the immersive training simulators in supporting training of medical residents in an orthopedic surgical procedure known as LISS plating surgical process. Next Generation Internet technologies explored include cloud principles and Software Defined Networking (SDN) to support interaction from distributed 
Table 6. QFS Results for Haptic and Immersive Simulator

\begin{tabular}{|l|c|c|}
\hline Criteria & $\begin{array}{c}\text { Immersive } \\
\text { Simulator }\end{array}$ & $\begin{array}{c}\text { Haptic } \\
\text { Simulator }\end{array}$ \\
\hline $\begin{array}{l}\text { 1. The user friendliness of the user interface of the menu } \\
\text { buttons and other interfaces. }\end{array}$ & 8.7 & 8.5 \\
\hline $\begin{array}{l}\text { 2. a. The effectiveness of the Vive Interface } \\
\text { b. Please give the specific training scenario where you } \\
\text { think the Vive interface has not been effective. }\end{array}$ & 8.6 & N/A \\
\hline $\begin{array}{l}\text { 3. a. The effectiveness of the haptic interface } \\
\text { b. Please give the specific training scenario where you } \\
\text { think the haptic interface has not been effective. }\end{array}$ & & 8.4 \\
\hline 4. The degree of user friendliness of the simulator. & 8.45 & 8.3 \\
\hline 5. The degree of usefulness of the text-based support. & 7.5 & 7.2 \\
\hline 6. The degree of usefulness of the 3D avatar support & 8.35 & 7.3 \\
\hline $\begin{array}{l}\text { 7. The ease of navigating through the simulation } \\
\text { environment (zooming, changing direction, performing } \\
\text { virtual walkthrough). }\end{array}$ & 7.9 & 4.2 \\
\hline $\begin{array}{l}\text { 8. Effort: How hard did you have to focus in order to } \\
\text { complete the training? }\end{array}$ & 4.1 & 2.5 \\
(1:Low, 10:High) & & 2.3 \\
\hline $\begin{array}{l}\text { 9. Frustration: How frustrated were you when trying to } \\
\text { complete the surgical steps in the training? }\end{array}$ & 2.45 & 2.1 \\
(1:Low, 10:High) & & 2.3 \\
\hline $\begin{array}{l}\text { 10. Mental Demand: How mentally demanding was the } \\
\text { training? } \\
\text { (1:Low, 10:High) }\end{array}$ & & \\
\hline $\begin{array}{l}\text { 11. Physical Demand: How physically demanding was the } \\
\text { training? } \\
\text { (1:Low, 10:High) }\end{array}$ & & \\
\hline $\begin{array}{l}\text { 12. Temporal Demand: How hurried or rushed were you } \\
\text { during the training? } \\
\text { (1:Low, 10:High) }\end{array}$ & & \\
\hline 13. Overall user experience in using the simulator & & \\
\hline
\end{tabular}

Table 7. Latency Data for On-Line Haptic Simulator

\begin{tabular}{|c|c|c|c|}
\hline \multirow{2}{*}{ Access } & \multicolumn{3}{|c|}{ Average Latency (ms) } \\
\cline { 2 - 4 } & User 1 & User 2 & User 3 \\
\hline 2 Users & 48.35 & 48.2 & N/A \\
\hline 3 Users & 48.7 & 48.5 & 48.9 \\
\hline
\end{tabular}

participants. The simulators' potential in training residents was assessed with the involvement of the Paul L. Foster School of Medicine at the Texas Tech Health Sciences Center (El Paso, Texas); a majority of participants showed significant improvements in their understanding of the LISS plating surgical process after interacting and learning how to use both simulators. User experience surveys rated the immersive VR-based simulator higher than the haptic-based simulator. 


\section{ACKNOWLEDGMENTS}

We would like to express our thanks to the surgeons, residents and students at the Paul L. Foster School of Medicine and the Texas Tech Health Sciences Center in El Paso (Texas) for participating in this project. The technical assistance received from Heidi Dempsey, Vicraj Thomas, Ali Sydney, and others at BBN Raytheon in GENI-networking-related implementation tasks is gratefully acknowledged.

\section{REFERENCES}

[1] B. Tolsdorff, A. Pommert, K. H. Höhne, A. Petersik, B. Pflesser, U. Tiede, and R. Leuwer. 2010. Virtual reality: A new paranasal sinus surgery simulator. The Laryngoscope, 120, 2, 420-426.

[2] K. S. Choi, S. Soo, and F. L. Chung. 2009. A virtual training simulator for learning cataract surgery with phacoemulsification. Computers in Biology and Medicine, 39, 11, 1020-1031.

[3] G. Echegaray, I. Herrera, I. Aguinaga, C. Buchart, and D. Borro. 2014. A brain surgery simulator. IEEE Computer Graphics and Applications, 34, 3, 12-18.

[4] C. Luciano, P. Banerjee, and T. DeFanti. 2009. Haptics-based virtual reality periodontal training simulator. Virtual Reality, 13, 2, 69-85.

[5] Y. Shi, Y. Xiong, X. Hua, K. Tan, and X. Pan. 2015. Key techniques of haptic related computation in virtual liver surgery. In 2015 8th International Conference on Biomedical Engineering and Informatics (BMEI). IEEE, 355-359.

[6] L. Yu, T. Wang, W. Wang, Z. Wang, and B. Zhang. 2013. A geometric modeling method based on OpenGL in virtual gallbladder surgery. In 2nd International Conference on Computer Science and Electronics Engineering. Atlantis Press.

[7] T. M. Peters, C. A. Linte, J. Moore, D. Bainbridge, D. L. Jones, and G. M. Guiraudon. 2008. Towards a medical virtual reality environment for minimally invasive cardiac surgery. In International Workshop on Medical Imaging and Virtual Reality. Springer, Berlin, 1-11.

[8] T. S. Sørensen, S. V. Therkildsen, P. Makowski, J. L. Knudsen, and E. M. Pedersen. 2001. A new virtual reality approach for planning of cardiac interventions. Artificial Intelligence in Medicine, 22, 3, 193-214.

[9] T. Berlage, A. Schmitgen, C. Schmitz, and A. Welz. 2001. Simulation and planning of minimally invasive coronary artery bypass surgery. In International Congress Series. Elsevier, 68-72.

[10] V. T. Le and S. Nahavandi. 2010. A haptic training environment for the heart myoblast cell injection procedure. In 11th International Conference on Control Automation Robotics \& Vision (ICARCV). IEEE, 448-452.

[11] J. Ren, R. V. Patel, K. A. McIsaac, G. Guiraudon, and T. M. Peters. 2008. Dynamic 3-D virtual fixtures for minimally invasive beating heart procedures. IEEE Transactions on Medical Imaging, 27, 8, 1061-1070.

[12] R. Yu, S. Zhang, P. Chiang, Y. Cai, and J. Zheng. 2010. Real-time and realistic simulation for cardiac intervention with GPU. In 2nd International Conference on Computer Modeling and Simulation (ICCMS'10). IEEE, 68-72.

[13] G. Azzie, J. T. Gerstle, A. Nasr, D. Lasko, J. Green, O. Henao, and A. Okrainec. 2011. Development and validation of a pediatric laparoscopic surgery simulator. Fournal of Pediatric Surgery, 46, 5, 897-903.

[14] S. A. Seixas-Mikelus, T. Kesavadas, G. Srimathveeravalli, R. Chandrasekhar, G. E. Wilding, and K. A. Guru. 2010. Face validation of a novel robotic surgical simulator. Urology, 76, 2, 357-360.

[15] K. Makiyama, M. Nagasaka, T. Inuiya, K. Takanami, M. Ogata, and Y. Kubota. 2012. Development of a patient specific simulator for laparoscopic renal surgery. International fournal of Urology, 19, 9, 829-835.

[16] T. P. Grantcharov, V. B. Kristiansen, J. Bendix, L. Bardram, J. Rosenberg, and P. Funch-Jensen. 2004. Randomized clinical trial of virtual reality simulation for laparoscopic skills training. British fournal of Surgery, 91, 2, 146-150.

[17] R. Aggarwal, I. Balasundaram, and A. Darzi. 2008. Training opportunities and the role of virtual reality simulation in acquisition of basic laparoscopic skills. Journal of Surgical Research, 145, 1, 80-86.

[18] J. Torkington, S. G. Smith, B. I. Rees, and A. Darzi. 2000. The role of simulation in surgical training. Annals of the Royal College of Surgeons of England, 82, 2, 88.

[19] A. Sourin, O. Sourina, and H. T. Sen. 2000. Virtual orthopedic surgery training. IEEE Computer Graphics and Applications, 20, 3, 6-9.

[20] J. Qin, W. M. Pang, Y. P. Chui, T. T. Wong, and P. A. Heng. 2010. A novel modeling framework for multilayered soft tissue deformation in virtual orthopedic surgery. Fournal of Medical Systems, 34, 3, 261-271.

[21] S. L. Delp and J. P. Loan. 1995. A graphics-based software system to develop and analyze models of musculoskeletal structures. Computers in Biology and Medicine, 25, 1, 21-34.

[22] M. D. Tsai, M. S. Hsieh, and S. B. Jou. 2001. Virtual reality orthopedic surgery simulator. Computers in Biology and Medicine, 31, 5, 333-351. 
[23] J. W. Park, J. Choi, Y. Park, and K. Sun. 2011. Haptic virtual fixture for robotic cardiac catheter navigation. Artificial Organs, 35, 11, 1127-1131.

[24] The American Board of Orthopedic Surgery. Retrieved from https://www.abos.org/abos-surgical-skills-modulesfor-pgy-1-residents.aspx.

[25] M. D. Tsai, C. S. Liu, H. Y. Liu, M. S. Hsieh, and F. C. Tsai. 2011. Virtual reality facial contouring surgery simulator based on CT transversal slices. In 5th International Conference on Bioinformatics and Biomedical Engineering (iCBBE). IEEE, 1-4.

[26] M. Vankipuram, K. Kahol, A. McLaren, and S. Panchanathan. 2010. A virtual reality simulator for orthopedic basic skills: A design and validation study. Fournal of Biomedical Informatics, 43, 5, 661-668.

[27] P. Blyth, N. S. Stott, and I. A. Anderson. 2007. A simulation-based training system for hip fracture fixation for use within the hospital environment. Injury, 38, 10, 1197-1203.

[28] S. Bayonat, M. García, C. Mendoza, and J. M. Fernandez. 2006. Shoulder arthroscopy training system with force feedback. In International Conference on Medical Information Visualisation-BioMedical Visualisation (MedVis'06). IEEE, 71-76.

[29] J. Petersson, K. L. Palmerius, H. Knutsson, O. Wahlstrom, B. Tillander, and M. Borga. 2008. Simulation of patient specific cervical hip fracture surgery with a volume haptic interface. IEEE Transactions on Biomedical Engineering, 55, 4, 1255-1265.

[30] H. Sabri, B. Cowan, B. Kapralos, M. Porte, D. Backstein, and A. Dubrowskie. 2010. Serious games for knee replacement surgery procedure education and training. Procedia-Social and Behavioral Sciences, 2, 2, 3483-3488.

[31] I. Kovler, L. Joskowicz, Y. A. Weil, A. Khoury, A. Kronman, R. Mosheiff, and J. Salavarrieta. 2015. Haptic computerassisted patient-specific preoperative planning for orthopedic fractures surgery. International fournal of Computer Assisted Radiology and Surgery, 10, 10, 1535-1546.

[32] M. D. Tsai, M. S. Hsieh, and C. H. Tsai. (2007). Bone drilling haptic interaction for orthopedic surgical simulator. Computers in Biology and Medicine, 37, 12, 1709-1718.

[33] O. Sourina, A. Sourin, and H. T. Sen. 2000. Virtual orthopedic surgery training on personal computer. International fournal of Information Technology, 6, 1, 16-29.

[34] M. X. Kong, Z. J. Du, L. N. Sun, L. X. Fu, Z. H. Jia, and D. M. Wu. 2006. A robot-assisted orthopedic telesurgery system. In 2005 27th Annual IEEE Conference Engineering in Medicine and Biology. IEEE, 97-101.

[35] L. Rosenblum M. Macedonia. 2000. Virtual orthopedic surgery training. IEEE Computer Graphics and Applications, 20, 3, 6-9.

[36] G. Burdea, V. Popescu, V. Hentz, and K. Colbert. 2000. Virtual reality-based orthopedic telerehabilitation. IEEE Transactions on Rehabilitation Engineering, 8, 3, 430-432.

[37] M. A. Padilla-Castaneda, E. Sotgiu, A. Frisoli, M. Bergamasco, P. Orsini, A. Martiradonna, and C. Laddaga. 2013. A virtual reality system for robotic-assisted orthopedic rehabilitation of forearm and elbow fractures. In 2013 IEEE/RSF International Conference on Intelligent Robots and Systems. IEEE, 1506-1511.

[38] M. Eriksson, H. Flemmer, and J. Wikander. 2005. Haptic simulation of the milling process in temporal bone operations. Studies in Health Technology and Informatics 111 (2005), 133-136.

[39] D. d'Aulignac, M. C. Cavusoglu, and C. Laugier. 1999. Modeling the dynamics of the human thigh for a realistic echographic simulator with force feedback. In International Conference on Medical Image Computing and ComputerAssisted Intervention. Springer, Berlin, 1191-1198.

[40] T. Seth, V. Chaudhary, C. Buyea, and L. Bone. 2011. A virtual interactive navigation system for orthopedic surgical interventions. In 4th International Symposium on Applied Sciences in Biomedical and Communication Technologies, ACM, 71.

[41] W. I. Willaert, R. Aggarwal, I. Van Herzeele, N. J. Cheshire, and F. E. Vermassen. 2012. Recent advancements in medical simulation: patient-specific virtual reality simulation. World fournal of Surgery, 36, 7, 1703-1712.

[42] C. Karaliotas. 2011. When simulation in surgical training meets virtual reality. Hellenic fournal of Surgery, 83, 6 , 303-316.

[43] J. P. Braman, R. M. Sweet, D. M. Hananel, P. M. Ludewig, and A. E. Van Heest. 2015. Development and validation of a basic arthroscopy skills simulator. Arthroscopy: The fournal of Arthroscopic \& Related Surgery, 31, 1, 104-112.

[44] C. Andersen, T. N. Winding, and M. S. Vesterby. 2011. Development of simulated arthroscopic skills: A randomized trial of virtual-reality training of 21 orthopedic surgeons. Acta orthopedica, 82, 1, 90-95.

[45] K. Rose and R. Pedowitz. 2015. Fundamental arthroscopic skill differentiation with virtual reality simulation. Arthroscopy: The fournal of Arthroscopic \& Related Surgery, 31, 2, 299-305.

[46] J. J. Stunt, G. M. M. J. Kerkhoffs, C. N. van Dijk, and G. J. M. Tuijthof. 2015. Validation of the arthros virtual reality simulator for arthroscopic skills. Knee Surgery, Sports Traumatology, Arthroscopy, 23, 11, 3436-3442.

[47] J. Qin, W. M. Pang, Y. P. Chui, T. T. Wong, and P. A. Heng. 2010. A novel modeling framework for multilayered soft tissue deformation in virtual orthopedic surgery. Fournal of Medical Systems, 34, 3, 261-271. 
[48] Y. Lin, X. Wang, F. Wu, X. Chen, C. Wang, and G. Shen. 2014. Development and validation of a surgical training simulator with haptic feedback for learning bone-sawing skill. fournal of Biomedical Informatics, 48, 122-129.

[49] NASA TLX Test. Retrieved from https://humansystems.arc.nasa.gov/groups/TLX/downloads/TLX.pdf.

[50] A. Nahvi, M. Moghaddam, M. Arbabtafti, M. Mahvash, and B. Richardson. 2016. Virtual bone surgery using a haptic robot. International fournal of Robotics, Theory and Applications, 1, 1, 1-12.

[51] M. Citak, M. J. Gardner, D. Kendoff, S. Tarte, C. Krettek, L. P. Nolte, and T. Hüfner. 2008. Virtual 3D planning of acetabular fracture reduction. Journal of Orthopedic Research, 26, 4, 547-552.

[52] L. Assassi, C. Charbonnier, J. Schmid, P. Volino, and N. Magnenat-Thalmann. 2009. From MRI to anatomical simulation of the hip joint. Computer Animation and Virtual Worlds, 20, 1, 53-66.

[53] Y. Jun and S. Park. 2011. Polygon-based 3D surgical planning system for hip operation. International fournal of Precision Engineering and Manufacturing, 12, 1, 157-160.

[54] D. Morris, C. Sewell, N. Blevins, F. Barbagli, and K. Salisbury. 2004. A collaborative virtual environment for the simulation of temporal bone surgery. In International Conference on Medical Image Computing and Computer-Assisted Intervention. Springer, Berlin Heidelberg, 319-327.

[55] B. Chebbi, D. Lazaroff, F. Bogsany, P. X. Liu, L. Ni, and M. Rossi. 2005. Design and implementation of a collaborative virtual haptic surgical training system. In IEEE International Conference Mechatronics and Automation. IEEE, 315-320.

[56] P. V. Paiva, L. D. S. Machado, A. M. G. Valença, R. M. De Moraes, and T. V. Batista. 2016. Enhancing collaboration on a cloud-based CVE for supporting surgical education. In 2016 XVIII Symposium on Virtual and Augmented Reality (SVR). IEEE, 29-36.

[57] S. W. Tang, K. L. Chong, J. Qin, Y. P. Chui, S. S. M. Ho, and P. A. Heng. 2007. ECiSS: A middleware based development framework for enhancing collaboration in surgical simulation. In IEEE International Conference on Integration Technology (ICIT'07). IEEE, 15-20.

[58] C. Jay, M. Glencross, and R. Hubbold. 2007. Modeling the effects of delayed haptic and visual feedback in a collaborative virtual environment. ACM Transactions on Computer-Human Interaction (TOCHI), 14, 2, 8.

[59] E. Acosta and A. Liu. 2007. Real-time interactions and synchronization of voxel-based collaborative virtual environments. In 2007 IEEE Symposium on 3D User Interfaces. IEEE.

[60] P. Youngblood, P. M. Harter, S. Srivastava, S. Moffett, W. L. Heinrichs, and P. Dev. 2008. Design, development, and evaluation of an online virtual emergency department for training trauma teams. Simulation in Healthcare, 3, 3, 146153.

[61] B. R. A. Sales, L. S. Machado, and R. M. Moraes. 2011. Interactive collaboration for virtual reality systems related to medical education and training. Technology and Medical Sciences, 157-162.

[62] J. C. De Oliveira and N. D. Georganas. 2003. VELVET: An adaptive hybrid architecture for very large virtual environments. Presence: Teleoperators and Virtual Environments, 12, 6, 555-580.

[63] J. Qin, K. S. Choi, W. S. Poon, and P. A. Heng. 2009. A framework using cluster-based hybrid network architecture for collaborative virtual surgery. Computer Methods and Programs in Biomedicine, 96, 3, 205-216.

[64] R. Caceres and A. Friday. 2012. Ubicomp systems at 20: Progress, opportunities, and challenges. IEEE Pervasive Computing, 11, 1, 14-21.

[65] C. Mégard, F. Gosselin, S. Bouchigny, F. Ferlay, and F. Taha. 2009. User-centered design of a maxillo-facial surgery training platform. In 16th ACM Symposium on Virtual Reality Software and Technology. ACM, 265-266.

[66] G. Brandt, A. Zimolong, L. Carrat, P. Merloz, H. W. Staudte, S. Lavallee, and G. Rau. 1999. CRIGOS: A compact robot for image-guided orthopedic surgery. IEEE Transactions on Information Technology in Biomedicine, 3, 4, $252-260$.

[67] J. Forsslund, E. L. Sallnas, and K. J. Palmerius. 2009. A user-centered designed FOSS implementation of bone surgery simulations. In EuroHaptics Conference, 2009 and Symposium on Haptic Interfaces for Virtual Environment and Teleoperator Systems. World Haptics 2009. IEEE, 391-392.

[68] A. Jalote-Parmar and P. Badke-Schaub. 2008. Workflow integration matrix: A framework to support the development of surgical information systems. Design Studies, 29, 4, 338-368.

[69] P. Jannin. 2013. Surgical process modeling: Methods and applications. In Presentation at the 2013 Medicine Meets Virtual Reality Conference (NEXTMED/MMVR20).

[70] A. Nemani, G. Sankaranarayanan, K. Roberts, L. Panait, C. Cao, and S. De. 2013. Hierarchical task analysis of hybrid rigid scope natural orifice translumenal endoscopic surgery (NOTES) cholecystectomy procedures. Studies in Health Technology and Informatics, 184, 293-297.

[71] J. Cecil, M. B. R. Kumar, A. Gupta, M. Pirela-Cruz, E. Chan-Tin, and J. Yu. 2016. Development of a virtual reality based simulation environment for orthopedic surgical training. In OTM Confederated International Conferences On the Move to Meaningful Internet Systems. Springer, 206-214.

[72] J. Cecil, A. Gupta, and M. Pirela-Cruz. 2018. An advanced simulator for orthopedic surgical training. International Journal of Computer Assisted Radiology and Surgery, 13, 2, 305-319. 
[73] Communication diagrams. Retrieved from http://agilemodeling.com/style/collaborationDiagram.htm.

[74] J. Cecil, A. Gupta, M. Pirela-Cruz, and P. Ramanathan. 2017. A cyber training framework for orthopedic surgery. Cogent Medicine, 4, 1, 1419792.

[75] GENI. Retrieved from www.geni.net.

[76] FIRE. Retrieved from https://www.ict-fire.eu/.

[77] M. Berman, J. S. Chase, L. Landweber, A. Nakao, M. Ott, D. Raychaudhuri, and I. Seskar. 2014. GENI: A federated testbed for innovative network experiments. Computer Networks, 61, 5-23.

[78] Software Defined Networking. Retrieved from https://www.opennetworking.org/sdn-resources/sdn-definition.

[79] S. Wijewickrema, B. Copson, Y. Zhou, X. Ma, R. Briggs, J. Bailey, G. Kennedy, and S. O’Leary. 2017. Design and evaluation of a virtual reality simulation module for training advanced temporal bone surgery. In 2017 IEEE 30 th International Symposium on Computer-Based Medical Systems (CBMS). IEEE, 7-12.

[80] James D. Watterson, Darren T. Beiko, James K. Kuan, and John D. Denstedt. 2002. A randomized prospective blinded study validating acquisition of ureteroscopy skills using a computer based virtual reality endourological simulator. The fournal of Urology, 168, 5, 1928-1932.

[81] Schlickum Marcus Kolga, Leif Hedman, Lars Enochsson, Ann Kjellin, and Li Felländer-Tsai. 2009. Systematic video game training in surgical novices improves performance in virtual reality endoscopic surgical simulators: A prospective randomized study. World fournal of Surgery, 33, 11, 2360.

[82] Steam VR toolkit. Retrieved from https://partner.steamgames.com/vrlicensing.

[83] VRTK. Retrieved from https://vrtoolkit.readme.io/.

[84] Vive. Retrieved from https://www.vive.com/us/.

[85] E. G. G. Verdaasdonk, J. Dankelman, J. F. Lange, and L. P. S. Stassen. 2008. Transfer validity of laparoscopic knottying training on a VR simulator to a realistic environment: A randomized controlled trial. Surgical Endoscopy, 22, 7, $1636-1642$.

[86] N. R. Howells, H. S. Gill, A. J. Carr, A. J. Price, and J. L. Rees. 2008. Transferring simulated arthroscopic skills to the operating theatre: A randomised blinded study. Bone \& foint fournal, 90, 4, 494-499.

Received August 2017; revised March 2018; accepted June 2018 